\title{
New signal representation based on the fractional Fourier transform: definitions
}

\author{
David Mendlovic, Zeev Zalevsky, Rainer G. Dorsch, and Yigal Bitran \\ Faculty of Engineering, Tel Aviv University, 69978 Tel Aviv, Israel
}

Adolf W. Lohmann

Department of Physics of Complex Systems, Weizmann Institute of Science, 76100 Rehovot, Israel

Haldun Ozaktas

Faculty of Electrical Engineering, Bilkent University, Turkey

Received January 25, 1995; revised manuscript received June 7, 1995; accepted June 27, 1995

\begin{abstract}
The fractional Fourier transform is a mathematical operation that generalizes the well-known Fourier transform. This operation has been shown to have physical and optical fundamental meanings, and it has been experimentally implemented by relatively simple optical setups. Based on the fractional Fourier-transform operation, a new space-frequency chart definition is introduced. By the application of various geometric operations on this new chart, such as radial and angular shearing and rotation, optical systems may be designed or analyzed. The field distribution, as well as full information about the spectrum and the space-bandwidth product, can be easily obtained in all the stages of the optical system. () 1995 Optical Society of America
\end{abstract}

\section{INTRODUCTION}

The name of this paper was originally "Linear phasespace representation based on the fractional Fourier transform"; however, we changed the title because the term phase space means different things to different people. We did not want to create wrong expectations, yet we feel that the main concept is still best explained if we use the loosely defined term "phase space," taking into account that, in the text below, phase-space representation means space-frequency representation.

Phase-space representations are useful tools for dual time-frequency analysis, image compression, data processing, and also designing and analyzing optical systems. For optical implementations, the well-known $Y \omega$ representation considers light as a bunch of rays, although each ray has a spatial location and direction (see Fig. 1). These two parameters are represented as a coordinate in the $Y \omega$ plane. Thus the $Y \omega$ chart has two axes, one for presenting the spatial coordinate $y$ and the other for the angle of the ray's propagation $\omega$, i.e., the derivation of the first coordinate. Note that two-dimensional rays are translated to a four-dimensional $Y \omega$ chart. One of the greatest disadvantages of such a representation is that the ray model does not take into account the diffraction effects; thus the output result is only a ray approximation of the light propagation.

Another type of phase-space representation is the Wigner transform. ${ }^{1}$ The Wigner transform is a mathematical operation applied to the input field distribution $f(x)$ :

$$
\begin{aligned}
\mathcal{W}[f(x)]= & W(x, \nu)=\int_{-\infty}^{\infty} f\left(x+\frac{x^{\prime}}{2}\right) f^{*}\left(x-\frac{x^{\prime}}{2}\right) \\
& \times \exp \left(-2 \pi i \nu x^{\prime}\right) \mathrm{d} x^{\prime} .
\end{aligned}
$$

This transform represents the spatial and the spectral properties of the function simultaneously, taking into account diffraction phenomena as well. However, this transform suffers from the fact that it is not linear (but bilinear). Thus sometimes it is not convenient to use this transform with linear systems.

Reconstruction of a function from its Wigner chart can be done with

$$
f(x)=\frac{1}{f^{*}(0)} \int_{-\infty}^{\infty} W\left(\frac{x}{2}, \nu\right) \exp (2 \pi i \nu x) \mathrm{d} \nu
$$

However, there is an uncertainty factor of a phase coefficient when the inverse Wigner transformation is performed.

For analyzing and synthesizing optical systems, the $Y \omega$ and the Wigner representations provide various promising properties. The elementary optical operators, freespace propagation and multiplication with a lens, are expressed as $X$-shearing and $Y$-shearing transformations (see Fig. 2), respectively, applied on the input field displayed in one of these phase-space representations. Thus these tools may overcome the intensive use of complicated chirp integrals in the optical design process, and both the field distribution and its spectrum can be obtained immediately from the phase-space presentation.

Below we present a novel phase-space representation that may be advantageous in various cases. We call this representation the $(r, p)$ chart. This representation can be used for designing optical systems and provides properties similar to those of the $Y \omega$ and Wigner representations. Also the field and its spectrum at different planes can be easily extracted, and elementary optical systems, such as free-space propagation or a lens, cause simple operations that we call radial shearing or angular shearing 


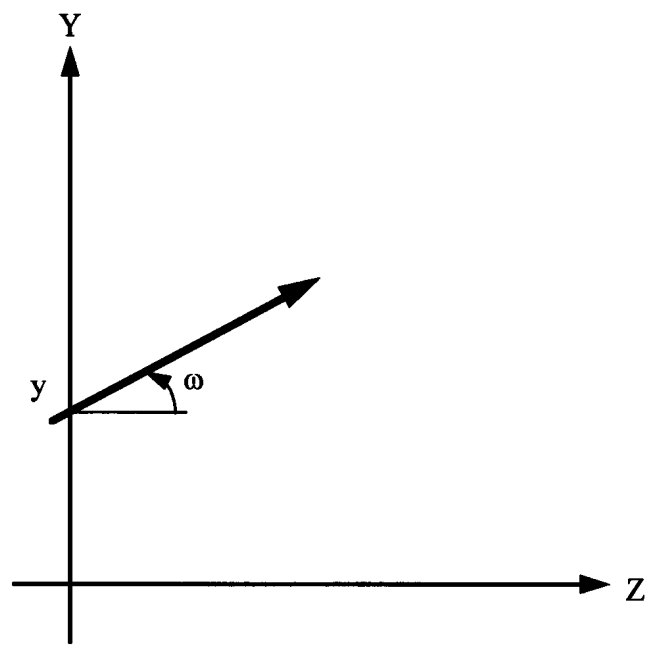

Fig. 1. Schematic illustration of the $Y \omega$ representation.
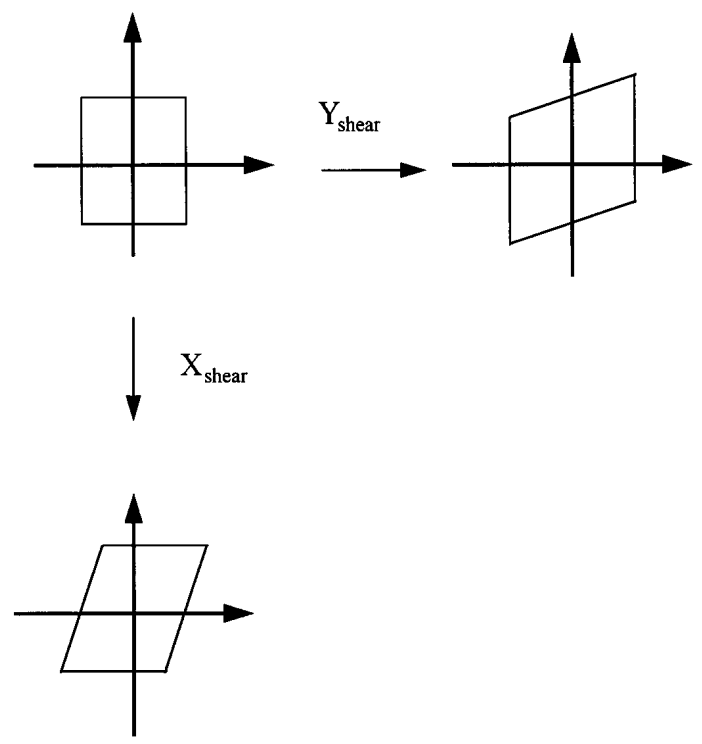

Fig. 2. Schematic illustration of $X$ - and $Y$-shearing.

of the new representation. Because it is a linear representation, it is easily reversible and it takes into account diffraction effects. As is shown in Section 2, this representation involves the fractional Fourier transform (FRT), a linear transformation that generalizes the conventional Fourier transform and that was recently introduced to the optics community.

Section 2 gives the relevant details about the FRT. Section 3 introduces the exact definition of the new representation, and Section 4 gives some of its mathematical properties, including the definition of the radial- and the angular-shearing operations.

An important note that is related to digital image processing and tomography applications of the FRT is the following. Recently, a new time-frequency analyzing tool, the Radon-Wigner transform, was suggested ${ }^{2,3}$ and used for the time-frequency representation of signals. ${ }^{4,5}$ Without being named, this approach led exactly to a chart that contains a continuous representation of the FRT of a signal as a function of the fractional Fourier order. As we mention below, we call this representation the $(x, p)$ chart, and it may also be useful in optics because it ex- plicitly shows the propagation of a signal inside a gradedindex (GRIN) medium.

\section{FRACTIONAL FOURIER TRANSFORM}

The FRT is a new mathematical tool to be used, for example, in spatial filtering operations. The Fourier transform of fractional order $p$ is defined in such a manner that the common Fourier transform is a special case with order $p=1$. An optical implementation of the FRT is provided in terms of quadratic GRIN media or in a setup that involves free-space propagation-lens-free-space propagation or lens-free-space propagation-lens.

There are two common definitions for the FRT. Both definitions were proven to be identical, as shown in Ref. 6 . The first optical FRT definition ${ }^{7-9}$ is modeled as the variation of the field during propagation along a quadratic GRIN medium by a length proportional to $p$, where $p$ is the FRT order.

The second definition is based on the Wigner distribution function. ${ }^{10}$ This is a complete signal description that displays time and frequency information simultaneously. , $11^{1,}$

Both definitions were generalized through a transformation kernel, as illustrated in Ref. 12:

$$
\left\{\mathcal{F}^{p}[u(x)]\right\}(x)=\int_{-\infty}^{\infty} B_{p}\left(x, x^{\prime}\right) u\left(x^{\prime}\right) \mathrm{d} x^{\prime}
$$

where $B_{p}\left(x, x^{\prime}\right)$ is the kernel of the transformation, and it is equal (according to the first definition) to

$$
\begin{aligned}
B_{p}\left(x, x^{\prime}\right)= & \sqrt{2} \exp \left[-\pi\left(x^{2}+x^{\prime 2}\right)\right] \sum_{n=0}^{\infty} \frac{i^{-p n}}{2^{n} n !} \\
& \times H_{n}(\sqrt{2 \pi} x) H_{n}\left(\sqrt{2 \pi} x^{\prime}\right),
\end{aligned}
$$

where $H_{n}$ is a Hermite polynomial of order $n$, or, according to the second definition,

$$
\begin{aligned}
B_{p}\left(x, x^{\prime}\right)= & \frac{\exp \left\{-i\left[\frac{\pi \operatorname{sgn}(\sin \phi)}{4}-\frac{\phi}{2}\right]\right\}}{|\sin \phi|^{1 / 2}} \\
& \times \exp \left(i \pi \frac{x^{2}+x^{\prime 2}}{\tan \phi}-2 i \pi \frac{x x^{\prime}}{\sin \phi}\right) .
\end{aligned}
$$

\section{DEFINITION}

The Fourier transform of fractional order $p$ is defined in a manner such that the classical Fourier transform is a special case with order $p=1$. An optical implementation of the FRT is possible with quadratic GRIN media and bulk optics implementation (see Section 2 for further details). The integral definition of the FRT is

$$
\begin{aligned}
u_{p}(x)= & C_{1} \int_{-\infty}^{\infty} u\left(x_{0}\right) \exp \left[\frac{i \pi}{\tan \phi}\left(x_{0}^{2}+x^{2}\right)\right] \\
& \times \exp \left(\frac{-2 \pi i}{\sin \phi} x x_{0}\right) \mathrm{d} x_{0} \\
\phi= & p(\pi / 2)
\end{aligned}
$$




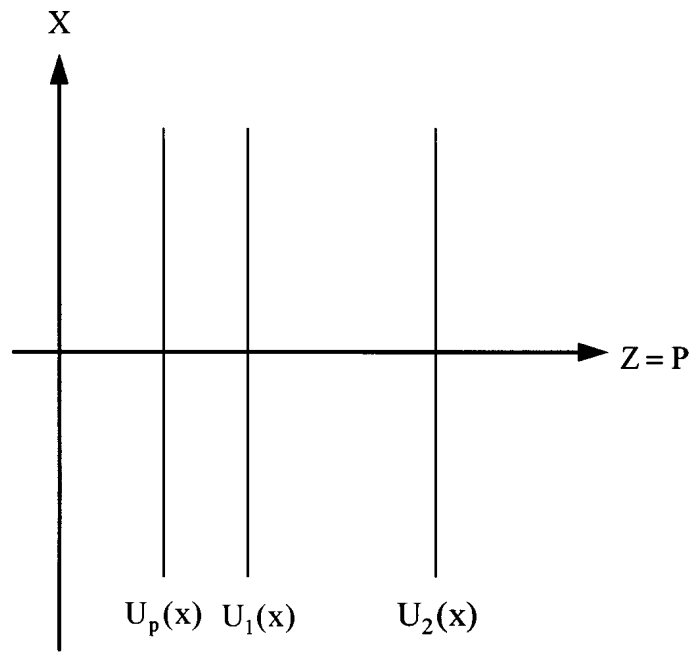

Fig. 3. Illustration of the $(x, p)$ chart.

where $p$ is the fractional order and $C_{1}$ is a normalizing constant that is given by

$$
C_{1}=\frac{\exp \left\{-i\left[\frac{\pi \operatorname{sgn}(\sin \phi)}{4}-\frac{\phi}{2}\right]\right\}}{|\sin \phi|^{1 / 2}} .
$$

One approach for presenting the different FRT orders with respect to the GRIN definition is a graphical chart that we call an $(x, p)$ chart. For a one-dimensional object this plot contains two axes. The vertical axis $x$ is the spatial one-dimensional light distribution $u_{p}(x)$ of the FRT order $p$ of the original function $u_{0}(x)$ [as given in Eqs. (6)]. The horizontal axis is the FRT order $p$ (see Fig. 3 for a graphical illustration). More explicitly, one can write

$$
F(x, p)=u_{p}(x)
$$

As a result, in this plot all the fractional Fourier orders of the original function $u_{0}(x)$ are calculated and displayed in one chart. Another point of view is that the $(x, p)$ chart explicitly shows the propagated light distribution within a GRIN piece. ${ }^{7-9}$ Note that the $(x, p)$ chart provides several interesting applications that will be described in a sequel to this paper.

The next step for obtaining what we call an $(r, p)$ chart is to perform a Cartesian-to-polar-coordinate transform of the $(x, p)$ chart. Here, all the fractional Fourier orders of the function are drawn as angular vectors. Each FRT order is drawn along the $r$ axis in specific angular orientation of $\phi=p(\pi / 2)$, where $p$ is the fractional order. Implicitly, one can write the $(r, p)$ representation as

$$
F(r, p)=u_{p}(r)
$$

Figure 4 gives a graphical illustration of the $(r, p)$ chart representation. It is important to note that $r$ may get negative values. The $r$ coordinate negative values are a by-product of the $(r, p)$ chart definition. However, discussing negative values for $r$ does not conflict with the polar-coordinate definition because

$$
u_{p+2}(r)=u_{p}(-r)
$$

Another note is connected with $r=0$. This singular point contains no relevant information and should be avoided when the chart is used. As a polar representation, the required spatial resolution for a lower $r$ value is higher. Thus, practically, a certain area of $|r|<r_{0}$ is not able to carry the necessary information (because of the limited spatial resolution of every plot) and must be avoided too. Figure 5 shows a schematic sketch of the interesting area of the $(r, p)$ chart. In this figure, $R_{\max }$ is the radius that confines the information of the fractional plane of the input object. The $(r, p)$ chart is our candidate for serving as a phase-space representation. It contains full information about the object (along $\phi=0$ ) and about its spectrum [along $\phi=(\pi / 2)$. Additional information regarding the mixture space-frequency information is given along with other values of $\phi$. The inverse transformation is trivial:

$$
u_{p}(r)=F(r, p),
$$

and, for the object itself,

$$
u_{0}(r)=F(r, 0) .
$$

In Section 4 several properties of the $(r, p)$ chart are analyzed and demonstrated.

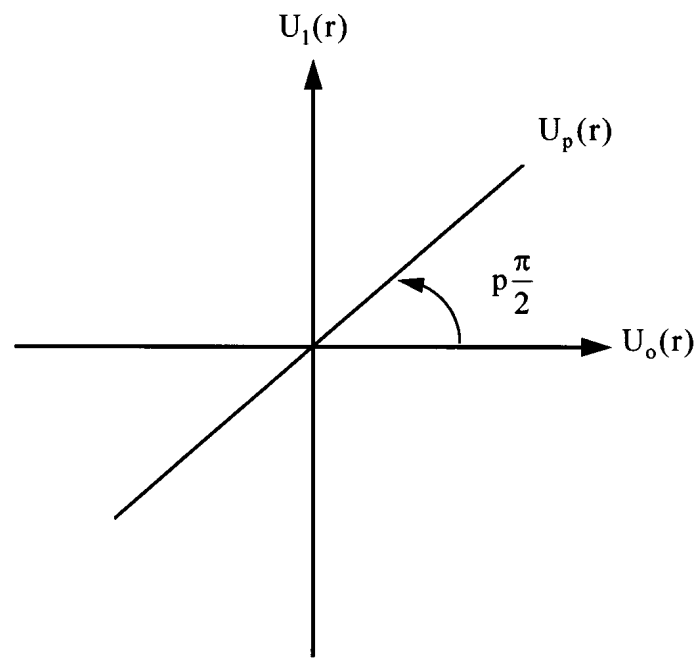

Fig. 4. Illustration of the $(r, p)$ chart.

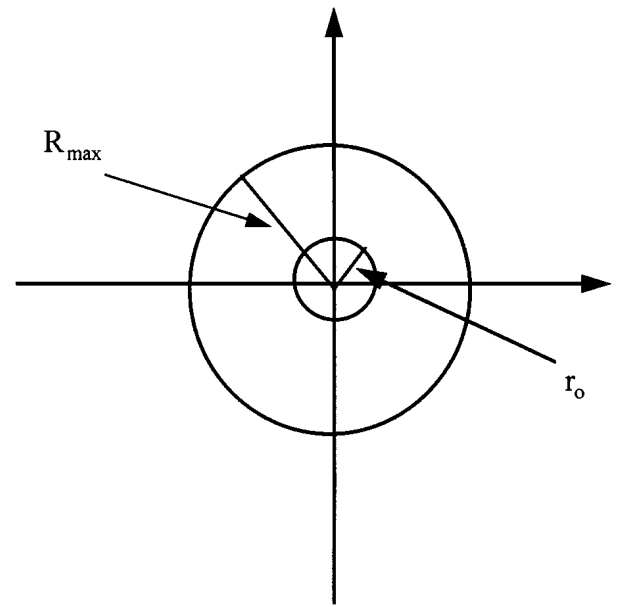

Fig. 5. Sketch of the interesting area in the $(r, p)$ chart. 


\section{MATHEMATICAL PROPERTIES}

A. Motivation

Let us recall from Lohmann ${ }^{10}$ that the FRT can be achieved by the following two algorithms:

Algorithm 1:

$$
\begin{aligned}
u\left(x_{0}\right) & \Rightarrow \mathcal{W}\left[u\left(x_{0}\right)\right]=W(x, \nu) \Rightarrow \operatorname{Rot}[W(x, \nu)] \\
& \Rightarrow \text { Inverse Wigner }=u_{p}(x) .
\end{aligned}
$$

Algorithm 2:

$$
\begin{aligned}
u\left(x_{0}\right) & \Rightarrow W(x, \nu) \Rightarrow X_{\text {shear }}[W(x, \nu)] \Rightarrow Y_{\text {shear }}[W(x, \nu)] \\
& \Rightarrow X_{\text {shear }}[W(x, \nu)] \Rightarrow \text { Inverse Wigner }=u_{p}(x)
\end{aligned}
$$

where Rot is the rotation operation in the plane. The shearing operations are defined as

$$
\begin{gathered}
X_{\text {shear }}[f(x, y)]=f(x+\alpha y, y), \\
Y_{\text {shear }}[f(x, y)]=f(x, y+\alpha x) .
\end{gathered}
$$

Because the lens operation in the Wigner plane is a $Y_{\text {shear }}$ and a free-space propagation is an $X_{\text {shear }}$, the procedure described in Eq. (14) is in fact a FRT operation.

Note that similar properties are relevant also for the $Y \omega$ diagram. The fact that the common optical operations (free-space propagation, lens, Fourier transform, and FRT) affect the Wigner and the $Y \omega$ in relatively simple geometric transformations increased the use of these charts for analyzing and synthesizing optical systems.

Our motivation is to show that the $(r, p)$ chart has similar properties and might be more suitable than the Wigner and the $Y \omega$ charts for some applications.

\section{B. Full Mathematical Definition}

The explicit mathematical definition of the $(r, p)$ chart is based on Eqs. (6) and (10) as follows:

$$
\begin{aligned}
F(r, p)=u_{p}(r)= & C_{1} \int_{-\infty}^{\infty} u\left(x_{0}\right) \exp \left(\pi i \frac{r^{2}+x_{0}^{2}}{\tan \phi}\right) \\
& \times \exp \left(-2 \pi i \frac{r x_{0}}{\sin \phi}\right) \mathrm{d} x_{0}
\end{aligned}
$$

For obtaining the conventional Fourier transform $(p=1)$, one should examine the distribution on the axis of $\phi=(\pi / 2)$ on the $(r, p)$ chart. More generally, to obtain any other FRT order $p$, one should examine the chart angular distribution at an angle of $(\pi p) / 2$.

Note that because the FRT definition is general enough to deal with all types of signal (complex as well), the information contained in the $(r, p)$ chart is not restricted to the type of signal.

\section{Fractional Fourier Transform Operation}

Assume a function $u\left(x_{0}\right)$ and its $(r, p)$ chart $F(r, p)$. The $(r, p)$ chart of $u_{q}\left(x_{0}\right)$ [FRT of order $q$ of $u\left(x_{0}\right)$ ] is

$$
F^{q}(r, p)=\left(u_{q}\right)_{p}(r)=u_{q+p}(r)=F(r, p+q) .
$$

One can note that $F^{q}(r, p)$ is a $(\pi q) / 2$ angular rotation of $F(r, p)$.
Thus one can conclude that performing a FRT means rotating the $(r, p)$ chart. Algorithm (13), based on the $(r, p)$ representation, is thus

$$
\begin{aligned}
u\left(x_{0}\right) & \Rightarrow F(r, p) \Rightarrow \operatorname{Rot}[F(r, p)] \\
& \Rightarrow \text { Inverse }(r, p) \text { chart } \Rightarrow u_{p}(x) .
\end{aligned}
$$

\section{Lens Operation}

One of the most common optical operators is a multiplication with a chirp function that represents a field distribution of $u_{0}\left(x_{0}\right)$ that passes through a lens. It can be written as $u_{0}\left(x_{0}\right) \exp \left(i \alpha^{\prime} \pi x_{0}{ }^{2}\right)$ when $\alpha^{\prime}$ is related to the lens focal length $f$ as

$$
\alpha^{\prime}=\frac{-1}{\lambda f},
$$

where $\alpha^{\prime}$ is a physical parameter given in units of inverse square meters. Because the mathematical formulation has no unit, in order to use the parameter $\alpha^{\prime}$ there we define $\alpha=n \alpha^{\prime}$, where $n=1\left(\mathrm{~m}^{2}\right)$.

Our interest is to find the effect on the $(r, p)$ chart with respect to the original chart $F(r, p)$. Let us denote the new $(r, p)$ chart as $F^{\text {lens }}(r, p)$. From Eqs. (6) one can note that

$$
\begin{aligned}
F^{\text {(lens })}(r, p) & \\
= & C_{1} \int_{-\infty}^{\infty} u_{0}\left(x_{0}\right) \exp \left(i \alpha \pi x_{0}{ }^{2}\right) \\
& \times \exp \left(i \pi \frac{x_{0}^{2}+r^{2}}{\tan \phi}\right) \exp \left(-i 2 \pi \frac{r x_{0}}{\sin \phi}\right) \mathrm{d} x_{0} \\
= & C_{1} \exp \left(i \pi \frac{r^{2}}{\tan \phi}\right) \int_{-\infty}^{\infty} \exp \left[i \pi x_{0}^{2}\left(\frac{1}{\tan \phi}+\alpha\right)\right] \\
& \times \exp \left(-i 2 \pi \frac{r x_{0}}{\sin \phi}\right) \mathrm{d} x_{0} .
\end{aligned}
$$

For simplicity, let us denote

$$
\beta=\frac{1}{\tan \phi}+\alpha=\frac{1}{\tan \theta} .
$$

From well-known trigonometric equations, one obtains

$$
\frac{1}{\sin \theta}=\sqrt{\beta^{2}+1} \text {. }
$$

Thus, based on the scale factor

$$
s=\frac{\sin \theta}{\sin \phi},
$$

Eq. (20) becomes

$$
\begin{aligned}
F^{(\text {lens })}(r, p)= & C_{1} \exp \left(i \pi \frac{r^{2}}{\tan \phi}\right) \int_{-\infty}^{\infty} u_{0}\left(x_{0}\right) \\
& \times \exp \left(i \pi \frac{x_{0}{ }^{2}}{\tan \theta}\right) \exp \left(-i 2 \pi x_{0} \frac{r s}{\sin \theta}\right) \mathrm{d} x_{0} \\
= & \psi u_{\theta}(r s)
\end{aligned}
$$

where $\psi$ is the quadratic phase factor outside the integral, and $u_{\theta}(r s)$ is the $(2 \theta) / \pi$ FRT order of the input function with a scale factor of $s$. As a result, one can note that the effect of a lens on the $(r, p)$ chart is a coordinate transformation. Each point inside the original chart is angularly rotated and radially scaled. The rotation $\theta-\phi$ 


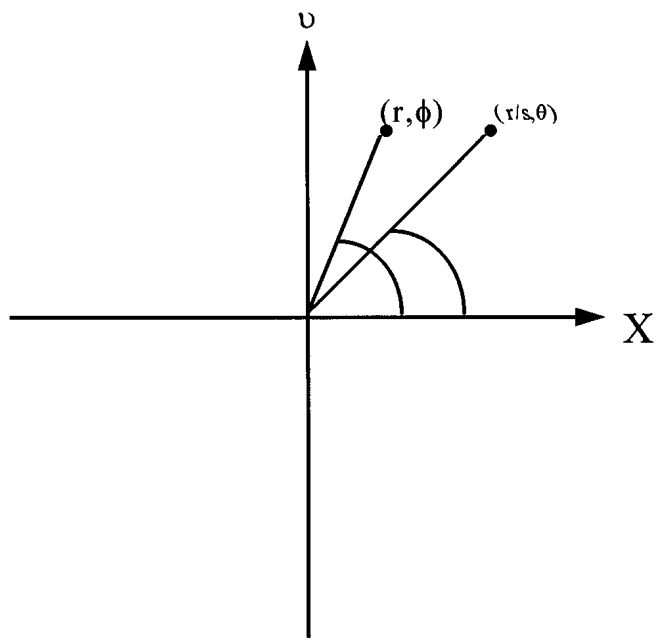

Fig. 6. Schematic illustration of the shear operation in polar coordinates.

and the scale $s$ are

$$
\begin{aligned}
\tan \theta & =\frac{\tan \phi}{1+\alpha \tan \phi}, \\
s & =\frac{1}{\sin \phi\left[\left(\frac{1}{\tan \phi}+\alpha\right)^{2}+1\right]^{1 / 2}} .
\end{aligned}
$$

We call this coordinate transformation a radial-shearing transformation. The motivation for this nickname is as follows. Let us examine what a conventional $X$-shearing is. Graphically, according to Eqs. (15), Fig. 6 represents an $X$-shearing operation example. After transformation to polar coordinates, Eqs. (15) become

$$
\begin{aligned}
& \theta=\tan ^{-1}\left(\frac{r \sin \phi}{r \cos \phi+\alpha r \sin \phi}\right), \\
& s=\frac{r}{\left[(r \sin \phi)^{2}+(r \cos \phi+\alpha r \sin \phi)^{2}\right]^{1 / 2}} .
\end{aligned}
$$

A division of the first equation of Eqs. (26) by $r \cos \phi$ and the second by $r$ leads to

$$
\begin{aligned}
& \theta=\tan ^{-1}\left(\frac{\tan \phi}{1+\alpha \tan \phi}\right), \\
& s=\frac{1}{\sin \phi\left[1+\left(\frac{1}{\tan \phi}+\alpha\right)^{2}\right]^{1 / 2}} .
\end{aligned}
$$

By inspection one can note that Eqs. (25) and (27) are exactly the same except that Eq. (25) relates to the scaled radius of $r s$ [Eq. (24)], and Eqs. (27) relate to $r / s$ (Fig. 6). Those rotation and scale factors are called the radial-shearing operation. Figures 7 and 8 are computer simulations that illustrate this new transformation operated on a square and on a circle, respectively. The radial-shearing transform was applied on two simple $(r, p)$ charts, a square and a circle. In Fig. 7(a) the original square is shown. Figures 7(b) and 7(c) show the transformed square according to regular $X$-shearing and radial-shearing operations, respectively. Figure 8(a) is a circled $F(r, p)$. Figures $8(\mathrm{~b})$ and 8(c) show again the regular $X$-shearing and the radial-shearing operations applied over this chart.

The regular $X$-shearing operation applied over a square turns the square into a parallelogram. When applied over a circle, the $X$-shearing operation turns the circle into an ellipse.

\section{E. Free-Space Propagation}

Another important optical operation is free-space propagation. According to the Fresnel integral, a signal $u_{0}\left(x_{0}\right)$ that propagates through the free space along a distance $z$ is

$$
u_{i}(x, z)=\frac{\exp \left(i \frac{2 \pi}{\lambda} z\right)}{i \lambda z} \int_{-\infty}^{\infty} u_{0}\left(x_{0}\right) \exp \left[\frac{i \pi}{\lambda z}\left(x_{0}-x\right)^{2}\right] \mathrm{d} x_{0} .
$$

One can note that the propagation integral is fully equivalent to a multiplication of the spectrum of $u_{0}\left(x_{0}\right)$ by $\exp \left(-i \pi \lambda z \nu^{2}\right)$, where $\nu$ is the frequency coordinate. Thus the free-space propagation is visualized as a rotation by $90^{\circ}$ of the $(r, p)$ chart, then a lens operation with $\alpha=$ $-m \lambda z$ [where $m=1\left(1 / m^{2}\right)$ because in our mathematical formulations we want $\alpha$ to be without units] is obtained, and then the $(r, p)$ chart is rotated back by $-90^{\circ}$. As a result, because we have already proved that the lens operation is analogous to an $X$-shearing operation and is called radial shearing, the $90^{\circ}$ rotation will force the free-space propagation to be analogous to the $Y$-shearing operation of the $(r, p)$ chart. This operation is called an angular-shearing operation.

\section{F. Space-Bandwidth Product Calculation}

So far we have investigated the effect of various optical operations on the $(r, p)$ chart. In this subsection we show another piece of information that can be extracted from the $(r, p)$ chart: the space-bandwidth product (SW) of the signal. In many cases, the knowledge of the SW is critical for the analysis and the design of optical systems. In general, obtaining the $\mathrm{SW}$ is relatively complicated and contains space and frequency calculations. Using the effect of a lens and free-space propagation on the $(r, p)$ chart, one can obtain the field distribution and the SW in every plane in the optical system. This ability gives the engineer a powerful tool for designing and analyzing optical systems.

The SW may be defined as

$$
\mathrm{SW}=\left(\Delta F_{0}\right)\left(\Delta F_{1}\right),
$$

where $\Delta F_{p}$ is the second moment of the function $F(r, p)$ at a specific value $p(\pi / 2)$, which is defined as

$$
\Delta F_{p}=\frac{\int_{-\infty}^{\infty} r^{2}|F(r, p)|^{2} \mathrm{~d} r}{\int_{-\infty}^{\infty}|F(r, p)|^{2} \mathrm{~d} r} .
$$

Hence, after each optical element, the $F(r, p)$ is recalculated (by the application of the radial- and the angularshearing and rotation operations), and with Eq. (29) the SW can be easily estimated, that is, the SW can be calculated at every plane of the optical system. The above 


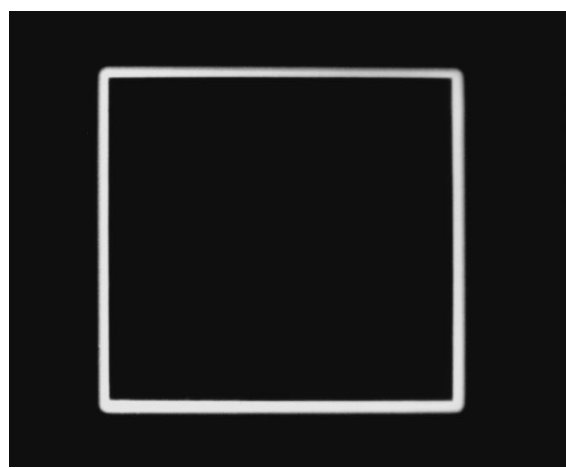

(a)

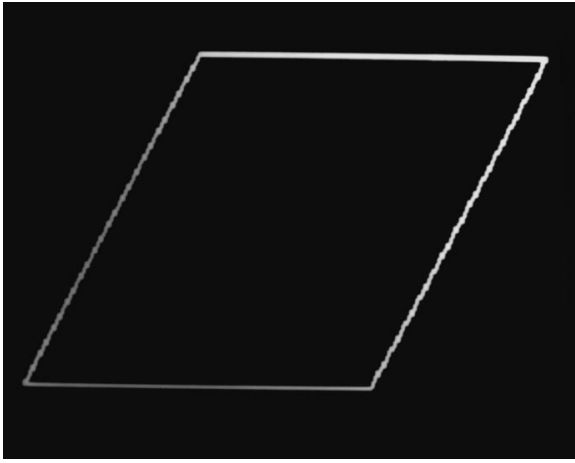

(b)

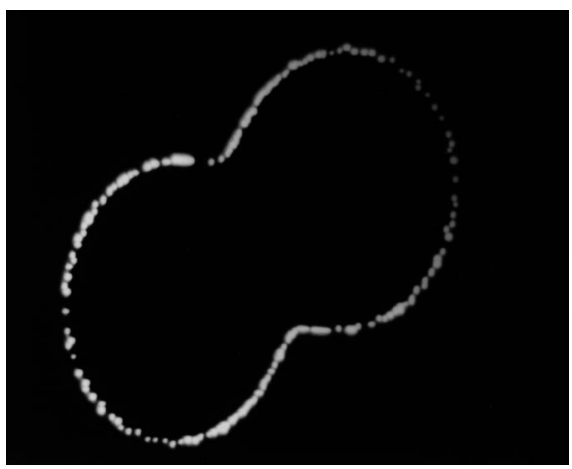

(c)

Fig. 7. (a) $(r, p)$ chart of a square, (b) its $X$-shearing transformation, (c) its radial-shearing transformation.

definition is for the SW of the signal itself. For finding the SW of other FRT orders, one can use the following equation:

$$
\mathrm{SW}(p)=\left(\Delta F_{p}\right)\left(\Delta F_{p+1}\right) .
$$

In several optical systems it is not necessary that $\mathrm{SW}(p)=\mathrm{SW}(0)$.

\section{G. Linearity}

The $F(r, p)$ chart is linear, which means that for two (or more) different signals $u_{0}\left(x_{0}\right)$ and $v_{0}\left(x_{0}\right)$, the associated $F(r, p)$ charts may be added:

$$
F_{\text {total }}(r, p)=\alpha F_{u}(r, p)+\beta F_{v}(r, p)
$$

where $F_{\text {total }}(r, p)$ refers to the chart of $\alpha u_{0}\left(x_{0}\right)+\beta v_{0}\left(x_{0}\right)$. This property does not exist in the Wigner transformation chart.

\section{H. Mathematical Validity}

In this subsection, several simple optical systems are tested with the $(r, p)$ chart in order to test the validity of the representation. We intend to show that elementary optical systems applied in cascade are equal to the relevant radial- or angular-shearing operation applied several times.

\section{Two Lenses in Cascade}

It was proved that a lens operation is a radial-shearing operation. Thus two lenses in cascade are equal to two radial-shearing operations applied one after the other. Let us assume that a lens with coefficient factor of $\alpha_{1}$ is applied. It has a certain radial-shearing effect on the $(r, p)$ chart. Then a second lens with another coefficient factor, $\alpha_{2}$, is applied, and again another radial shearing of the $(r, p)$ chart is obtained. Here we prove that applying one lens with total coefficient factor of $\alpha_{1}+\alpha_{2}$ causes a

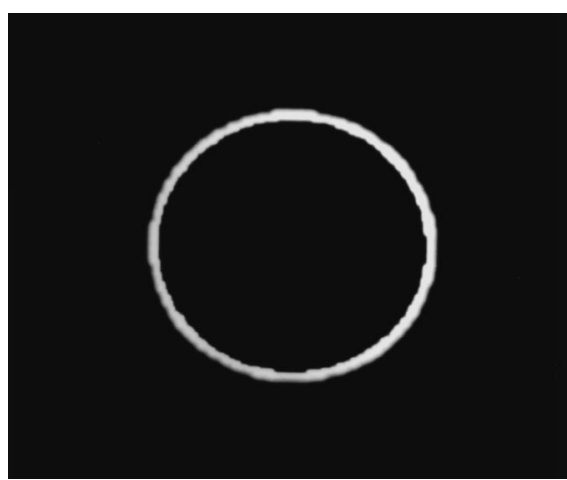

(a)

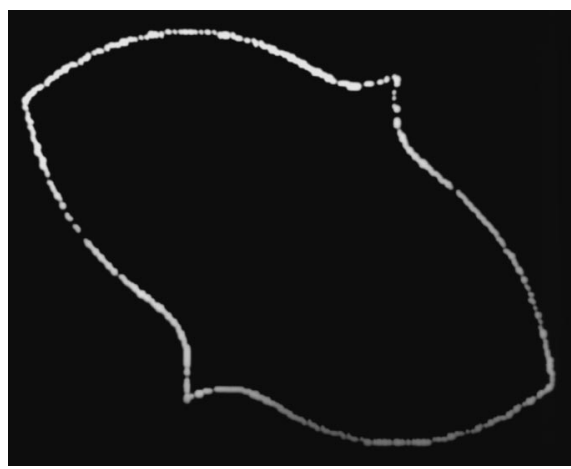

(b)

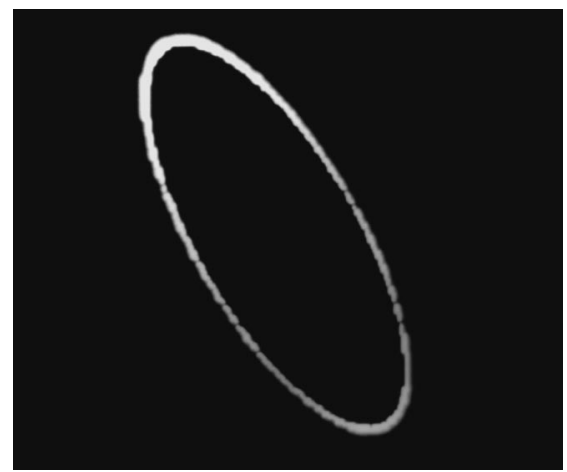

(c)

Fig. 8. Same as Fig. 7 but with an $(r, p)$ chart of a circle. 
radial shearing that is equal to the overall radial shearing obtained above in the two-stage operation.

A lens with a chirp factor of $\alpha_{1}+\alpha_{2}$ provides a radial shearing of

$$
\begin{aligned}
& \theta=\tan ^{-1}\left[\frac{\tan \phi}{1+\left(\alpha_{1}+\alpha_{2}\right) \tan \phi}\right], \\
& s=\frac{1}{\sin \phi\left\{\left[\frac{1}{\tan \phi}+\left(\alpha_{1}+\alpha_{2}\right)\right]^{2}+1\right\}^{1 / 2}} .
\end{aligned}
$$

On the other hand, applying two lens operations in cascade gives

$$
\begin{gathered}
(r, \phi) \Rightarrow\left(s_{1} r, \theta_{1}\right), \\
\left(s_{1} r, \theta_{1}\right) \Rightarrow\left(s_{2} s_{1} r, \theta\right),
\end{gathered}
$$

where

$$
\begin{aligned}
\theta_{1} & =\tan ^{-1}\left(\frac{\tan \phi}{1+\alpha_{1} \tan \phi}\right), \\
s_{1} & =\frac{1}{\sin \phi\left[\left(\frac{1}{\tan \phi}+\alpha_{1}\right)^{2}+1\right]^{1 / 2}}, \\
\theta & =\tan ^{-1}\left(\frac{\tan \theta_{1}}{1+\alpha_{2} \tan \theta_{2}}\right), \\
s_{2} & =\frac{1}{\sin \theta_{1}\left[\left(\frac{1}{\tan \theta_{1}}+\alpha_{2}\right)^{2}+1\right]^{1 / 2}} .
\end{aligned}
$$

After applying simple trigonometric equations such as

$$
\sin ^{2} \beta=\frac{\tan ^{2} \beta}{1+\tan ^{2} \beta},
$$

and after substituting them into Eqs. (35) one obtains exactly Eqs. (33) from expressions (34) and Eqs. (35) when $s=s_{1} s_{2}$

\section{Rotation}

As was mentioned above, FRT's may be obtained by the use of a bulk optics system that contains lens-freespace-lens operations. We intend to show that applying the three relevant shearing operations provides exactly a rotation ${ }^{10}$ of the $(r, p)$ chart. This may be expected from Subsection 4.C in which we show that a FRT means a rotation of the $(r, p)$ chart.

A regular shearing operation applied over $x$, then over $y$, and again over $x$, with factors of $A, B$, and $C$, is equivalent to

$$
\begin{aligned}
& \left(x_{0}, y_{0}\right) \Rightarrow\left(x_{0}-A y_{0}, y_{0}\right)=\left(x_{1}, y_{1}\right), \\
& \left(x_{1}, y_{1}\right) \Rightarrow\left(x_{1}, y_{1}+B x_{1}\right)=\left(x_{2}, y_{2}\right), \\
& \left(x_{2}, y_{2}\right) \Rightarrow\left(x_{2}-C y_{2}, y_{2}\right)=\left(x_{3}, y_{3}\right) .
\end{aligned}
$$

For obtaining a rotation by $\gamma$, the shearing coefficients should be

$$
A=C=\tan \frac{\gamma}{2}, \quad B=\sin \gamma .
$$

Now let us perform three modified shearing operations with factors of $\alpha, \beta$, and again $\alpha$, assuming that the same relation as in Eqs. (38) should be kept between the factors of the modified shearing, i.e., between $\alpha$ and $\beta$.

A modified shearing operation that is performed three times means that

$$
\begin{aligned}
(r, \phi) & \Rightarrow\left(s_{1} r, \theta_{1}\right), \\
\left(s_{1} r, \theta_{1}\right) & \Rightarrow\left(s_{1} r, \theta_{2}\right), \\
\left(s_{1} r, \theta_{2}\right) & \Rightarrow\left(s_{2} s_{1} r, \theta_{3}\right), \\
\left(s_{2} s_{1} r, \theta_{3}\right) & \Rightarrow\left(s_{2} s_{1} r, \theta_{4}\right), \\
\left(s_{2} s_{1} r, \theta_{4}\right) & \Rightarrow\left(s_{3} s_{2} s_{1} r, \theta_{5}\right),
\end{aligned}
$$

where

$$
\begin{aligned}
& \theta_{1}=\tan ^{-1}\left(\frac{\tan \phi}{1+\alpha \tan \phi}\right), \\
& \theta_{2}=\theta_{1}+(\pi / 2), \\
& \theta_{3}=\tan ^{-1}\left(\frac{\tan \theta_{2}}{1+\beta \tan \theta_{2}}\right), \\
& \theta_{4}=\theta_{3}-(\pi / 2), \\
& \theta_{5}=\tan ^{-1}\left(\frac{\tan \theta_{4}}{1+\alpha \tan \theta_{4}}\right), \\
& s_{1}=\frac{\sin \phi\left[\left(\frac{1}{\tan \phi}+\alpha\right)^{2}+1\right]^{1 / 2}}{\sin \theta_{2}\left[\left(\frac{1}{\tan \theta_{2}}+\beta\right)^{2}+1\right]^{1 / 2}}, \\
& s_{2}=\frac{1}{\sin \theta_{4}\left[\left(\frac{1}{\tan \theta_{4}}+\alpha\right)^{2}+1\right]^{1 / 2}} .
\end{aligned}
$$

Note that one performs the angular-shearing operation by first rotating the chart by $90^{\circ}$, then applying the radialshearing operation, and finally again rotating the chart by $-90^{\circ} . \quad \alpha$ is the radial-shearing factor, and $\beta$ is the angular-shearing factor. According to Eqs. (38) and to the trigonometric relation of

$$
\tan \frac{\gamma}{2}=\frac{\sin \gamma}{1+\left(1-\sin ^{2} \gamma\right)^{1 / 2}},
$$

one can obtain that

$$
\beta=\frac{2 \alpha}{\alpha^{2}+1} .
$$

Because

$$
\begin{aligned}
& \frac{1}{\tan \theta_{4}}=-\tan \theta_{3}, \\
& \sin \theta_{4}=-\cos \theta_{3}, \\
& \frac{1}{\tan \theta_{2}}=-\tan \theta_{1}, \\
& \sin \theta_{2}=\cos \theta_{1},
\end{aligned}
$$

and using the relations of Eqs. (40)-(42) and the trigonometric relation 


$$
\tan \left(\gamma_{1}+\gamma_{2}\right)=\frac{\tan \gamma_{1}+\tan \gamma_{2}}{1-\tan \gamma_{1} \tan \gamma_{2}},
$$

one obtains that

$$
\begin{aligned}
\theta_{5} & =\phi-\tan ^{-1} \frac{2 \alpha}{1-\alpha^{2}}, \\
s_{3} s_{2} s_{1} r & =r
\end{aligned}
$$

thus the radius $r$ is unchanged, and the angle is changed by $-\tan ^{-1}\left(2 \alpha / 1-\alpha^{2}\right)$, which is exactly the definition of rotation.

\section{CONCLUSIONS}

A novel, FRT-based, phase-space representation has been introduced. It was suggested as a powerful tool for designing and analyzing optical systems. The representation was coined the $(r, p)$ chart. This stage of drawing the $(r, p)$ chart involves several heavy calculations, but after this stage, the design information is complete, and in order to design or to analyze the optical system, the calculations that one should perform are negligible, because the shearing operation can be ultrafast when calculated by the computer (the amount of calculations is much smaller compared with the amount of calculations involved in algorithms such as fast Fourier transforms).

The system we design or analyze may consist of elementary optical elements such as free-space propagation, lenses, GRIN media, etc. Each such element causes a simple operation on the $(r, p)$ chart. The operation is a radial or an angular shearing. The calculation time required for obtaining such an operation is negligible. Using the inverse formula [Eq. (12)], one can easily obtain the field distribution at every stage of the optical system. Moreover, at each plane, the SW or the total energy of that plane can be calculated. All this can be done by the use of only the $(r, p)$ chart.

Because the novel phase chart representation is linear, is reversible, takes into account the diffraction effects, and delivers such useful information required for optical system design and analysis, we believe it can be a commonly used tool in electro-optics.

\section{ACKNOWLEDGMENT}

A. W. Lohmann acknowledges support from the Weizmann Institute of Science as a Michael visiting professor. A. W. Lohmann and R. G. Dorsch are on leave from Physikalisches Institute, Erlangen University, Erlangen, Germany.

\section{REFERENCES}

1. E. Wigner, "On the quantum correction for thermodynamics equilibrium,” Phys. Rev. 40, 749 (1932).

2. J. C. Wood and D. T. Barry, "Radon transform of the Wigner spectrum," in Advanced Signal Processing Algorithms, Architectures, and Implementations III, F. T. Luk, ed., Proc. Soc. Photo-Opt. Instrum. Eng. 1770, 358-375 (1992).

3. A. W. Lohmann and B. H. Soffer, "Relationships between the Radon-Wigner and fractional Fourier transforms," J. Opt. Soc. Am. A 11, 1798-1801 (1994).

4. J. C. Wood and D. T. Barry, "Linear signal synthesis using the Radon-Wigner transform," IEEE Trans. Acoust. Speech Signal Process. 42, 2105-2111 (1994).

5. J. C. Wood and D. T. Barry, "Tomographic time-frequency analysis and its application toward time-varying filtering and adaptive kernel design for multicomponent linear-FM signals," IEEE Trans. Acoust. Speech Signal Process. 42, 2094-2104 (1994).

6. D. Mendlovic, H. M. Ozaktas, and A. W. Lohmann, "Gradedindex fibers, Wigner distribution functions, and the fractional Fourier transform," Appl. Opt. 33, 6188-6193 (1994).

7. D. Mendlovic and H. M. Ozaktas, "Fractional Fourier transformations and their optical implementation: part I," J. Opt. Soc. Am. A 10, 1875-1881 (1993).

8. H. M. Ozaktas and D. Mendlovic, "Fractional Fourier transformations and their optical implementation: part II," J. Opt. Soc. Am. A 10, 2522-2531 (1993).

9. H. M. Ozaktas and D. Mendlovic, "Fourier transforms of fractional orders and their optical interpretation," Opt. Commun. 101, 163-165 (1993).

10. A. W. Lohmann, "Image rotation, Wigner rotation, and the fractional Fourier transform," J. Opt. Soc. Am. A 10, 2181-2186 (1993).

11. M. J. Bastiaans, "The Wigner distribution function applied to optical signals and systems," Opt. Commun. 25, 26-30 (1978).

12. H. M. Ozaktas, B. Barshan, D. Mendlovic, and L. Onural, "Convolution, filtering, and multiplexing in fractional Fourier domain and their relation to chirp and wavelet transforms," J. Opt. Soc. Am. A 11, 547-559 (1994). 